\title{
ON THE RANGE OF THE DERIVATIVE OF A SMOOTH MAPPING BETWEEN BANACH SPACES
}

ROBERT DEVILLE

Received 15 January 2004

We survey recent results on the structure of the range of the derivative of a smooth mapping $f$ between two Banach spaces $X$ and $Y$. We recall some necessary conditions and some sufficient conditions on a subset $A$ of $\mathscr{L}(X, Y)$ for the existence of a Fréchet differentiable mapping $f$ from $X$ into $Y$ so that $f^{\prime}(X)=A$. Whenever $f$ is only assumed Gâteaux differentiable, new phenomena appear: for instance, there exists a mapping $f$ from $\ell^{1}(\mathbb{N})$ into $\mathbb{R}^{2}$, which is bounded, Lipschitz-continuous, and so that for all $x, y \in \ell^{1}(\mathbb{N})$, if $x \neq y$, then $\left\|f^{\prime}(x)-f^{\prime}(y)\right\|>1$.

\section{Introduction}

The purpose of this work is to survey recent results obtained on the structure of the set of derivatives of a smooth function. Let $X, Y$ be separable Banach spaces such that $\operatorname{dim}(X) \geq 1$ and let $f: X \rightarrow Y$ be a mapping differentiable (in a sense to be specified later) at every point of $X$. We are interested in the structure of the range of the derivative of $f$, that is, in the set $f^{\prime}(X)=\left\{f^{\prime}(x) ; x \in X\right\} \subset \mathscr{L}(X, Y)$. Several notions of differentiability can be considered. We say that $f$ is Gâteaux differentiable at $x \in X$ provided that there exists $T \in \mathscr{L}(X, Y)$ such that for each $h \in X$,

$$
\lim _{t \rightarrow 0} \frac{f(x+t h)-f(x)}{t}=T(h),
$$

$T$ is called the Gâteaux derivative of $f$ at $x$ and is denoted $T=f^{\prime}(x)$. We say that $f$ is Fréchet differentiable at $x \in X$ provided that there exists $T \in \mathscr{L}(X, Y)$ such that for each $h \in X$,

$$
\lim _{\|h\| \rightarrow 0} \frac{f(x+h)-f(x)-T(h)}{\|h\|}=0
$$

$T$ is called the Fréchet derivative of $f$ at $x$ and is denoted $T=f^{\prime}(x)$. We are interested in the following questions.

(i) What are the topological properties of the set $f^{\prime}(X)$ ? 
(ii) For which sets $A \subset \mathscr{L}(X, Y)$ does there exist a smooth mapping $f: X \rightarrow Y$ such that $A=f^{\prime}(X)$ ? Smoothness of $f$ may have several meanings: $\mathscr{C}^{1}$ mapping, everywhere Fréchet differentiable mapping, everywhere Gâteaux differentiable mapping, and everywhere Fréchet differentiable mapping with Lipschitz continuous derivative. How does the notion of smoothness affect the answer to the above question?

(iii) What is the interplay between the geometry of the Banach spaces $X$ and $Y$ and the structure of the set $f^{\prime}(X)$ ? In particular, what additional properties of sets of the form $f^{\prime}(X)$ can be derived when $Y=\mathbb{R}$ ?

(iv) What additional properties can be obtained when $f$ has a nonempty bounded support (we then say that $f$ is a bump function)?

We will see in Section 2 necessary conditions on a set $A \subset X^{*}$ so that there exists a smooth function $f$ from $X$ into $\mathbb{R}$ such that $A=f^{\prime}(X)$. These necessary conditions depend on the kind of smoothness considered, and on the fact that $X$ is finite or infinite dimensional. We will give sufficient conditions so that there exists a $\mathscr{C}^{1}$ function $f$ from $X$ into $\mathbb{R}$ such that $A=f^{\prime}(X)$.

We present two results on the connectedness of $f^{\prime}(X)$ in Section 3 for real-valued functions. Easy counterexamples show that there is no hope of extending these results to mappings from a Banach space $X$ to a Banach space $Y$ of dimension greater than 1 .

We will present in Sections 4 and 5 phenomena which can occur when $f$ is Gâteaux differentiable, but not when $f$ is Fréchet differentiable, and which are detailed in [7]. In particular, for each infinite-dimensional separable Banach space $X$, we will construct in Section 4 a Gâteaux differentiable function $f$ on $X$, with bounded support, and such that for all $x \neq 0,\left\|f^{\prime}(x)-f^{\prime}(0)\right\| \geq 1$. However, given a Lipschitz and Gâteaux differentiable function $f$ from an arbitrary Banach space $X$ into $\mathbb{R}$, one can find, for every $\varepsilon>0$, two points $x, y \in X$ such that $\left\|f^{\prime}(x)-f^{\prime}(y)\right\|$ is less than $\varepsilon$. And if moreover $f$ has bounded nonempty support, then $f^{\prime}(X)$ contains a norm neighborhood of 0 in $X^{*}$. In Section 5, we will consider the following question. Let $X, Y$ be two Banach spaces. Is it possible to construct a Lipschitz continuous mapping $f: X \rightarrow Y$, Gâteaux differentiable at each point, and such that, for all $x, y \in X, x \neq y$, we have $\left\|f^{\prime}(x)-f^{\prime}(y)\right\| \geq 1$ ? Clearly, this is not possible whenever $\mathscr{L}(X, Y)$ is separable. We will prove that this is not possible either whenever $Y=\mathbb{R}$, but such a construction will be carried out whenever $(X, Y)=\left(\ell^{1}, \mathbb{R}^{2}\right)$ and whenever $(X, Y)=\left(\ell^{p}, \ell^{q}\right)$ with $1 \leq p \leq q<+\infty$.

\section{Sets which are the ranges of a derivative}

We are interested here in the study of the set

$$
\mathscr{S}=\left\{f^{\prime}(X) ; f \text { is a } \mathscr{b}^{1} \text {-bump on } X\right\} \text {. }
$$

Observe that if $X$ is separable with nonseparable dual, then there is no $\mathscr{C}^{1}$-smooth bump on $X$. So we will study $\mathscr{S}$ only for spaces with separable duals.

Elements of $\mathscr{Y}$ can be very small: it follows from [12] that if $f$ is a function on $c_{0}$ with locally uniformly continuous derivative, then $f^{\prime}\left(c_{0}\right)$ is included in a countable union of norm compact subsets of $\ell^{1}$. In particular, the norm-interior of $f^{\prime}\left(c_{0}\right)$ is empty in $\ell^{1}$. 
In [3], Azagra and Jiménez-Sevilla constructed an example of a $\mathscr{b}^{1}$-smooth function on $\ell^{2}$ so that the norm interior of $f^{\prime}\left(\ell^{2}\right)$ is empty in $\ell^{2}$.

Elements of $\mathscr{Y}$ can be very large: it was noticed in [1] that whenever $X$ is an infinitedimensional Banach space with separable dual, there exists a $\mathscr{C}^{1}$-smooth real-valued function on $X$ with bounded support and such that $f^{\prime}(X)=X^{*}$. On the other hand, it was observed in [4] that if $X$ and $Y$ are separable Banach spaces and if $X$ is infinite dimensional, one can always find a Gâteaux differentiable function $f: X \rightarrow Y$ such that $f^{\prime}(X)$ coincides with $\mathscr{L}(X, Y)$.

We recall that sufficient conditions on a subset $A$ of a dual Banach space $X^{*}$ so that it is the range of the derivative of a real-valued function on $X$ which is Fréchet differentiable at each point have been obtained in $[2,5,6,10]$. We recall here the results of [2].

In order to study $\mathscr{S}$ in finite-dimensional Banach spaces, we first introduce some definitions. Let $A \subset X^{*}$ be arcwise connected, and let $x, y \in A$. We define a distance in $A$ by the following formula:

$$
d_{A}(x, y)=\inf \{\operatorname{diam}(\gamma([0,1])) ; \gamma:[0,1] \longrightarrow A \text { continuous, } \gamma(0)=x, \gamma(1)=y\}
$$

and a measure of precompactness of $A$ for the distance $d_{A}$ is defined by the following indices:

$$
r_{n}(A)=\sup _{\left(y_{1}, y_{2}, \ldots, y_{n}\right) \in A^{n}}\left\{\inf \left\{d_{A}\left(y_{i}, y_{j}\right) ; 1 \leq i<j \leq n\right\}\right\}
$$

It is easy to see that whenever $X$ is finite dimensional, an element of $\mathscr{Y}$ is compact, arcwise connected, and 0 lies in its interior. Azagra, Fabian, and Jiménez-Sevilla proved the following theorem.

Theorem 2.1. Let $X$ be a finite-dimensional Banach space, and let $U \subset X^{*}$ be open, bounded, and connected such that $0 \in U$. Then,

$$
\lim _{n} r_{n}(U)=0 \Longrightarrow \bar{U} \in \mathscr{Y} \Longrightarrow \lim _{n} r_{n}(\bar{U})=0 .
$$

The structure of $\mathscr{Y}$ for infinite-dimensional Banach spaces has been investigated by [2], and then improved in [9]. Recall that a metric space $A$ is said to be analytic if there exists a mapping $\varphi: \mathbb{N}^{\mathbb{N}} \rightarrow A$ which is continuous and onto. Whenever $X$ is infinite dimensional, an element of $\mathscr{S}$ is analytic, arcwise connected, and 0 lies in the interior of its norm closure.

Theorem 2.2. Let $X$ be an infinite-dimensional Banach space with separable dual, and let $U \subset X^{*}$ be open and connected such that $0 \in U$. Let $A$ be an analytic subset of $X^{*}$ such that $U \subset A \subset \bar{U}$. Consider the following assertions:

(1) for all $x \in A$, there exists $\gamma:[0,1] \rightarrow$ A continuous, such that $\gamma(0)=0, \gamma(1)=x$, and $\gamma([0,1[) \subset U$,

(2) $A \in \mathscr{Y}$,

(3) for all $x \in A$, there exists $\gamma:[0,1] \rightarrow A$ continuous, such that $\gamma(0)=0$ and $\gamma(1)=x$. Then $(1) \Rightarrow(2) \Rightarrow(3)$. 
The structure of the range of $f^{\prime}$ whenever $f^{\prime}$ is Lipschitz continuous has been investigated in [11]. We denote

$$
\mathscr{S}_{1}=\left\{f^{\prime}(X) ; f \text { is a } \mathscr{C}^{1,1} \text {-bump on } X\right\} .
$$

Note that the existence of a $\mathscr{C}^{1,1}$-smooth bump on $X$ is a strong condition on $X$. It implies that $X$ is superreflexive. This condition is satisfied for instance in $L^{p}$ spaces for $2 \leq p<$ $+\infty$.

The condition $A \in \mathscr{S}_{1}$ is very restrictive in finite dimensions, as shown by the following necessary condition obtained by Gaspari. We first introduce some definitions. Let $A \subset X^{*}$ be arcwise connected, and let $x, y \in A$. We define a distance in $A$ by the following formula:

$$
\delta_{A}(x, y)=\inf \{\text { length }(\gamma([0,1])) ; \gamma:[0,1] \longrightarrow A \text { continuous, } \gamma(0)=x, \gamma(1)=y\},
$$

and a measure of precompactness of $A$ for the distance $\delta_{A}$ is defined by the following indices:

$$
\ell_{n}(A)=\sup _{\left(y_{1}, y_{2}, \ldots, y_{n}\right) \in A^{n}}\left\{\inf \left\{\delta_{A}\left(y_{i}, y_{j}\right) ; 1 \leq i<j \leq n\right\}\right\} .
$$

Proposition 2.3. Assume that $\operatorname{dim}(X)=d<+\infty$ and that $A \in \mathscr{I}_{1}$, then the sequence $\left(n^{1 / d} \ell_{n}(A)\right)$ is bounded.

We now give a sufficient condition on a set $A$ so that $A \in \mathscr{I}_{1}$. We say that a subset $A$ of $X^{*}$ is star shaped if for every $y^{*} \in A,\left[0, y^{*}\right] \subset A$. We say that $A$ is uniformly star shaped if there exists $\gamma>0$ such that for every $y^{*} \in A, \operatorname{conv}\left(\gamma B_{X^{*}} \cup\left\{y^{*}\right\}\right) \subset A$. Gaspari observed in [11] that there exists a compact star shaped subset of $\mathbb{R}^{d}$ such that 0 is in the interior of $K$ and $K \notin \mathscr{S}_{1}$, and there exists a bounded open star shaped subset $A$ of the separable infinite-dimensional Hilbert space so that $A \notin \mathscr{Y}^{1}$. However, he obtained the following theorem.

Theorem 2.4. Assume that $X$ is an infinite-dimensional Banach space so that there exists a $\mathscr{C}^{1,1}$-smooth bump function on $X$. If $U \subset X^{*}$ is open, bounded, and uniformly star shaped, then $U \in \mathscr{S}_{1}$.

\section{Is the range of $f^{\prime}$ connected?}

Whenever $f: X \rightarrow \mathbb{R}$ is everywhere Fréchet differentiable, this question was answered by J. Malý in 1996.

Theorem 3.1. If $X$ is a Banach space and $f: X \rightarrow \mathbb{R}$ is Fréchet differentiable at every point, then the set $f^{\prime}(X)$ is connected in $\left(X^{*},\|\cdot\|\right)$.

There exists a mapping $f$ from $\mathbb{R}^{2}$ into $\mathbb{R}^{2}$, Fréchet differentiable at each point, and so that the cardinal of $\left\{\operatorname{det}\left(f^{\prime}(x)\right) ; x \in \mathbb{R}^{2}\right\}$ is 2 . Therefore $f^{\prime}\left(\mathbb{R}^{2}\right)$ is not connected. Consequently, there is no analog of Malýs theorem for vector-valued mappings. Such an example was communicated to us by J. Saint-Raymond: take $f(x, y)=\left(x^{2} \sqrt{y} \cos 1 / x^{3}\right.$, $\left.x^{2} \sqrt{y} \sin 1 / x^{3}\right)$ whenever $(x, y) \neq(0,0)$ and $f(0,0)=(0,0)$. In this case, we have $\left\{\operatorname{det}\left(f^{\prime}(x)\right)\right.$; $\left.x \in \mathbb{R}^{2}\right\}=\{0,3 / 2\}$. 
Whenever $f$ is assumed to be Gâteaux differentiable, the following result was obtained by R. Deville and P. Hajek.

Proposition 3.2. Let $X$ be an infinite-dimensional Banach space, and let $f$ be a real-valued locally Lipschitz and Gâteaux differentiable function on $X$. Then either $f$ is affine or $f^{\prime}(X)$ has no $w^{*}$-isolated points.

This result was improved by Mátrai who recently obtained it in [13].

Proposition 3.3. Let $X$ be a separable Banach space, and let $f$ be a real-valued locally Lipschitz and Gâteaux differentiable function on $X$. Then $f^{\prime}(X)$ is connected in $\left(X^{*}, w^{*}\right)$.

\section{Isolated points in the range of the derivative of a function}

From now on, we say that a real-valued function on an infinite-dimensional Banach space $X$ is a bump function if it has bounded nonempty support. We will denote by $B(r)$ the set of all $x^{*} \in X^{*}$ such that $\left\|x^{*}\right\|<r$. If $X$ is a Banach space, $x \in X$, and $r>0$, we denote by $B_{X}(x, r)$ (resp., $\left.\bar{B}_{X}(x, r)\right)$ the open ball (resp., closed ball) in $X$ of center $x$ and radius $r$.

Proposition 4.1. If $f$ is a continuous and Gâteaux differentiable bump function on $X$, then the norm closure of $f^{\prime}(X)$ contains a ball $B(r)$ for some $r>0$.

Proof. The ranges of the derivative of $f$ and of the function $a f\left(\left(\cdot-x_{0}\right) / a\right)$ are the same. So, there is no loss of generality if we assume that $f(0) \neq 0$ and the support of $f$ is contained in the unit ball. Furthermore, if the conclusion of the proposition holds for $f$, it also holds for $-f$. So we can assume that $f(0)<0$. Let $g \in X^{*}$ be such that $\|g\|<$ $-f(0)$, fix $\varepsilon$ arbitrary such that $0<\varepsilon<-f(0)-\|g\|$, and define $\varphi(x)=f(x)-g(x)$ if $x \leq 1$ and $\varphi(x)=+\infty$ otherwise. According to the Ekeland variational principle, there exists $x_{0}$ such that $\varphi(x) \geq \varphi\left(x_{0}\right)-\varepsilon\left\|x-x_{0}\right\|$. We have $\left\|x_{0}\right\|<1$, otherwise $\left\|x_{0}\right\|=1$ and $\varphi(0)=f(0) \geq \varphi\left(x_{0}\right)-\varepsilon\left\|x_{0}\right\| \geq\|g\|-\varepsilon$, a contradiction with the choice of $\varepsilon$. Therefore $\left\|\varphi^{\prime}\left(x_{0}\right)\right\|=\left\|f^{\prime}\left(x_{0}\right)-g\right\| \leq \varepsilon$. This shows that the norm closure of $f^{\prime}(X)$ contains the ball $B(-f(0))$.

In view of the above proposition, a natural conjecture would be that the norm closure of $f^{\prime}(X)$ is norm connected, or at least that $f^{\prime}(X)$ does not contain an isolated point. This is not so as shown by the following construction.

Theorem 4.2. Let $X$ be an infinite-dimensional separable Banach space. Then, there exists a bump function $f$ on $X$ such that $f$ is Gâteaux differentiable at every point, $f^{\prime}$ is norm-toweak ${ }^{*}$ continuous, and $\left\|f^{\prime}(0)-f^{\prime}(x)\right\| \geq 1$ whenever $x \neq 0$. If $X^{*}$ is separable, it can be assumed that $f$ is $\mathscr{C}^{1}$ on $X \backslash\{0\}$.

Remark 4.3. According to the above discussion, 0 is not an isolated point of $f^{\prime}(X)$, so necessarily $f^{\prime}(0) \neq 0$.

Proof. The theorem is proved using the following two lemmas.

Lemma 4.4. Let $X$ be a Banach space, let $U$ be an open connected subset of $X^{*}$ such that $0 \in U$ and $x^{*} \in U$. Assume that there exists on $X$ a Lipschitz continuous bump function which is Gâteaux differentiable (resp., Fréchet differentiable) at every point. Then there exists 
a Lipschitz continuous bump function $\beta$ on $X$ with support contained in the unit ball, which is Gâteaux differentiable (resp., Fréchet differentiable) at every point, such that $\beta^{\prime}(X) \subset U$ and $\beta^{\prime}(x)=x^{*}$ for all $x$ in a neighborhood of 0 .

Lemma 4.5. Let $X, Y$ be two Banach spaces, $a \in X$, let $V$ be an open neighborhood of $a$, and let $f: V \rightarrow Y$ be continuous on $V$ and Gâteaux differentiable at every point of $V \backslash\{a\}$. If $f^{\prime}(x)$ has a limit $\ell$ in $\mathscr{L}(X, Y)$ endowed with the strong operator topology as $x$ tends to $a$, then $f$ is Gâteaux differentiable at $a$ and $f^{\prime}(a)=\ell$.

Sketch of proof of Theorem 4.2. Let $a^{*} \in X^{*}$ such that $1<\left\|a^{*}\right\|<2$. It is possible to construct $\left(W_{n}\right)_{n \geq 0}$, a decreasing sequence of norm open, norm connected, and weak ${ }^{*}$ open subsets so that

(1) if $y_{n}^{*} \in W_{n}$ and if $\left(y_{n}^{*}\right)$ is bounded, then $\left(y_{n}^{*}\right)$ converges to $a^{*}$ for the weak ${ }^{*}$ topology,

(2) for every $n$ and every $x \in W_{n},\left\|x-a^{*}\right\|>1$.

Let $\left(x_{n}^{*}\right) \subset X^{*}$ be a sequence such that $x_{1}^{*}=0$ and for every $n, x_{n}^{*} \in W_{n}$. Since $X$ is separable (resp., $X^{*}$ is separable), there exists on $X$ a Lipschitz continuous bump function which is Gâteaux differentiable (resp., Fréchet differentiable) at each point. According to Lemma 4.4, there exists a Lipschitz continuous bump $b_{n}$ which is Gâteaux differentiable (resp., Fréchet differentiable) at every point, such that $b_{n}^{\prime}(X) \subset W_{n}-x_{n}^{*}$, with support in the unit ball and such that $b_{n}^{\prime}(x)=x_{n+1}^{*}-x_{n}^{*}$ for all $x$ satisfying $\|x\|<\delta_{n}$. Denote $c_{1}=1$ and for $n \geq 2, c_{n}=\prod_{i=1}^{n-1} \delta_{n}$. Define

$$
b(x)=\sum_{n=1}^{+\infty} c_{n} b_{n}\left(\frac{x}{c_{n}}\right)
$$

$b$ is Gâteaux differentiable at each point of $X \backslash\{0\}$, and its support is contained in the unit ball. By construction, $b^{\prime}(X \backslash\{0\}) \subset X^{*} \backslash B\left(a^{*}, 1\right)$, and $b^{\prime}(x) \stackrel{w^{*}}{\longrightarrow} a^{*}$ as $x \rightarrow 0$. Lemma 4.5 then shows that $b$ is Gâteaux differentiable at 0 and that $b^{\prime}(0)=a^{*}$.

\section{Can all the derivatives be far away from each other?}

The aim of this section is to investigate the following question: if $X, Y$ are Banach spaces, is it possible to construct a mapping $f: X \rightarrow Y$, everywhere differentiable, so that for every $x, y \in X$, if $x \neq y$, then $\left\|f^{\prime}(x)-f^{\prime}(y)\right\| \geq 1$ ? We first notice that, under mild regularity assumptions, the answer to the above question is negative for real-valued functions.

Proposition 5.1. Let $X$ be a Banach space and let $f: X \rightarrow \mathbb{R}$ be a Lipschitz continuous (or merely locally uniformly continuous), everywhere Gâteaux differentiable function. Then, for every $x \in X$ and every $\varepsilon>0$, there exist $y, z \in B_{X}(x, \varepsilon)$ such that $\left\|f^{\prime}(y)-f^{\prime}(z)\right\| \leq \varepsilon$.

Idea of proof. Take any $h \in X$ such that $\|h\|$ is small enough, and consider the lower semicontinuous function defined by $\varphi(y)=f(y+h)-f(y)$. The Ekeland variational principle then tells us the existence of $y \in X$, not far from $x$ such that $\left\|\varphi^{\prime}(y)\right\| \leq \varepsilon$. Therefore, if we denote $z=y+h$, then $\left\|f^{\prime}(y)-f^{\prime}(z)\right\| \leq \varepsilon$, and $y$ and $z$ are not far from $x$.

The answer to the above question will also be negative if $f$ is everywhere Fréchet differentiable. 
Proposition 5.2. Let $X, Y$ be separable Banach spaces and let $f: X \rightarrow Y$ be an everywhere Fréchet differentiable locally uniformly continuous mapping. Then, for every $x \in X$ and every $\varepsilon>0$, there exist $y, z \in B_{X}(x, \varepsilon), y \neq z$, such that $\left\|f^{\prime}(y)-f^{\prime}(z)\right\| \leq \varepsilon$.

The answer to the above question will also be negative if $\mathscr{L}(X, Y)$ is separable.

Proposition 5.3. Let $X, Y$ be Banach spaces and let $f: X \rightarrow Y$ be an everywhere Gâteaux differentiable function. If $\mathscr{L}(X, Y)$ is separable, then, for every $x \in X$ and every $\varepsilon>0$, there exist $y, z \in B_{X}(x, \varepsilon)$ such that $\left\|f^{\prime}(y)-f^{\prime}(z)\right\| \leq \varepsilon$.

In view of the above propositions, one could believe that whenever $X, Y$ are $\mathrm{Ba}$ nach spaces (or vector-normed spaces) and $f: X \rightarrow Y$ is a mapping which is Gâteaux differentiable at each point of $X$, then for every $\varepsilon>0$, there exist $y, z \in X$ such that $\left\|f^{\prime}(y)-f^{\prime}(z)\right\| \leq \varepsilon$. Our next result proves that this is not so.

Theorem 5.4. There exists a Lipschitz mapping $F: \ell^{1} \rightarrow \mathbb{R}^{2}$, Gâteaux differentiable at each point of $\ell^{1}$, such that $\left\|F^{\prime}(x)-F^{\prime}(y)\right\|_{\mathscr{L}\left(\ell^{1}, \mathbb{R}^{2}\right)} \geq 1$ whenever $x, y \in \ell^{1}, x \neq y$. Moreover, for each $h \in \ell^{1}, x \rightarrow F^{\prime}(x) h$ is continuous from $\ell^{1}$ into $\mathbb{R}^{2}$.

We will construct $F$ and $G$ with the properties of Theorem 5.4 using series. We were inspired by a construction from [8]. We need an auxiliary construction.

Lemma 5.5. Given $\Delta=\left(a^{\prime}, a, b, b^{\prime}\right) \in \mathbb{R}^{4}$ such that $a^{\prime}<a<b<b^{\prime}$ and $\varepsilon>0$, there exists $a$ $\mathscr{C}^{\infty}$-function $\varphi=\varphi_{\Delta, \varepsilon}: \mathbb{R}^{2} \rightarrow \mathbb{R}^{2}$ such that

(i) $|\varphi(x, y)| \leq \varepsilon$ for all $(x, y) \in \mathbb{R}^{2}$,

(ii) $\varphi(x, y)=0$ whenever $x \notin\left[a^{\prime}, b^{\prime}\right]$,

(iii) $\|(\partial \varphi / \partial x)(x, y)\| \leq \varepsilon$ for all $(x, y) \in \mathbb{R}^{2}$,

(iv) $\|(\partial \varphi / \partial y)(x, y)\|=1$ whenever $x \in[a, b]$,

(v) $\|(\partial \varphi / \partial y)(x, y)\| \leq 1$ for all $(x, y) \in \mathbb{R}^{2}$,

(vi) if $\varphi(x, y)=\left(\varphi_{1}(x, y), \varphi_{2}(x, y)\right)$, then $\left(\partial \varphi_{1} / \partial y\right)(x, 0)=1$ whenever $x \in[a, b]$.

Proof of Lemma 5.5. Let $\beta: \mathbb{R} \rightarrow \mathbb{R}$ be a $\mathscr{C}^{\infty}$-smooth function such that $0 \leq \beta(x) \leq 1$ for all $x, \beta(x)=0$ whenever $x \notin\left[a^{\prime}, b^{\prime}\right]$, and $\beta(x)=1$ whenever $x \in[a, b]$. If $n \geq 1$ is large enough, then the function defined by $\varphi(x, y)=(\beta(x) / n)(\sin (n y), \cos (n y))$ satisfies the desired properties.

We will also use the following criterion of Gâteaux differentiability of the sum of a series.

Gâteaux differentiability criterion. Let $X$ and $Y$ be Banach spaces and, for all $n$, let $f_{n}$ : $X \rightarrow Y$ be Gâteaux differentiable mappings. Assume that $\left(\sum f_{n}\right)$ converges pointwise on $X$, and that

$$
\forall h,\left(\sum \frac{\partial f_{n}}{\partial h}(x)\right) \text { converges uniformly with respect to } x \text {. }
$$

Then the mapping $f=\sum_{n \geq 1} f_{n}$ is Gâteaux differentiable at every point of $X$, and for all $x \in X, f^{\prime}(x)=\sum_{n \geq 1} f_{n}^{\prime}(x)$ (where the convergence of the series is in $\mathscr{L}(X, Y)$ for the strong operator topology). 
The proof of this criterion is elementary and omitted here.

Sketch of proof of Theorem 5.4. Fix an enumeration $\Delta_{k}=\left(a_{k}^{\prime}, a_{k}, b_{k}, b_{k}^{\prime}\right), k \in N$, of all quadruples of dyadic numbers such that $a_{k}^{\prime}<a_{k}<b_{k}<b_{k}^{\prime}$. Fix $m_{k}^{n}=2^{k} \cdot 3^{n}, \varepsilon>0$, and let $\varepsilon_{k}^{n}$ be positive real numbers such that $\sum_{n=1}^{\infty} \sum_{k=1}^{\infty} \varepsilon_{k}^{n}=\varepsilon$. Define $f_{n, k}: \ell^{1} \rightarrow \mathbb{R}^{2}$ such that if $x=\left(x_{i}\right) \in \ell^{1}$, then

$$
f_{n, k}(x)=\varphi_{\Delta_{k}, \varepsilon_{k}^{n}}\left(x_{n}, x_{m_{k}^{n}}\right) .
$$

$f_{n, k}$ is a $\mathscr{C}^{\infty}$ mapping on $\ell^{1}$. Define $F: \ell^{1} \rightarrow \mathbb{R}^{2}$ by

$$
F(x)=\sum_{n \in \mathbb{N}} \sum_{k \in \mathbb{N}} f_{n, k}(x)
$$

It is easy to see that $F$ is well defined. One can also check that for all $h$,

$$
\sum_{n \geq 1} \sup _{x \in X}\left\|\frac{\partial f_{n}}{\partial h}(x)\right\| \leq(1+\varepsilon)\|h\| .
$$

So, for all $h,\left(\sum\left(\partial f_{n} / \partial h\right)(x)\right)$ converges uniformly with respect to $x$. According to the Gâteaux differentiability criterion, the mapping $F$ is Gâteaux differentiable on $\ell^{1}$, and $F$ is $(1+\varepsilon)$-Lipschitz-continuous on $\ell^{1}$. Finally, the mappings $f_{n, k}$ have been selected in such a way that if $x \neq y \in \ell^{1}$, then $\left\|F^{\prime}(x)-F^{\prime}(y)\right\|_{\mathscr{L}\left(\ell^{1}, \mathbb{R}^{2}\right)} \geq 1-2 \varepsilon$.

Remark 5.6. Note that for $p>1$, the dual of $\ell^{P}$ is separable. Therefore, by Proposition 5.3, it is not possible to replace $\ell^{1}$ by $\ell^{p}(p>1)$ in Theorem 5.4. However, there exists a Lipschitz function $H: \ell^{2} \rightarrow \ell^{2}$, Gâteaux differentiable at each point of $\ell^{2}$, such that for every $x, y \in \ell^{2}$, if $x \neq y$, then

$$
\left\|H^{\prime}(x)-H^{\prime}(y)\right\|_{\mathscr{L}\left(\ell^{2}\right)} \geq 1 .
$$

This will follow from the following more general result.

Theorem 5.7. Let $X_{p}=\ell^{p}$ if $1 \leq p<+\infty$ and $X_{\infty}=c_{0}$. Fix $1 \leq p, q \leq+\infty$. The following assertions are equivalent.

(1) There exists a Lipschitz continuous mapping $H: X_{p} \rightarrow X_{q}$, Gâteaux differentiable at each point of $X_{p}$, such that for every $x, y \in X_{p}, x \neq y$, then $\left\|H^{\prime}(x)-H^{\prime}(y)\right\|_{\mathscr{L}\left(X_{p}, X_{q}\right)} \geq 1$.

(2) $p \leq q$.

(3) $\mathscr{L}\left(X_{p}, X_{q}\right)$ is not separable.

Proof of Theorem 5.7. According to Proposition 5.3 above, (1) implies (3). If $p>q$, then by Pitt's theorem, all operators from $X_{p}$ to $X_{q}$ are compact, hence $\mathscr{L}\left(X_{p}, X_{q}\right)$ is separable. Therefore (3) implies (2). So it remains to prove that (2) implies (1). Assume that $p \leq q$ and let $\left(e_{n}\right)$ be the usual basis of $X_{p}$. Let $T_{k} \in \mathscr{L}\left(\mathbb{R}^{2}, X_{q}\right)$ defined by $T_{k}(x, y)=x e_{2 k}+$ $y e_{2 k+1}$. Let $\Delta_{k}, \varepsilon_{k}^{n}, m_{k}^{n}$, and $\varphi_{\Delta_{k}, \varepsilon_{k}^{n}}$ be defined as in the proof of Theorem 5.4. Let $f_{n, k}$ : $X_{p} \rightarrow X_{q}$ be such that if $x=\left(x_{i}\right) \in X_{p}$, then $f_{n, k}(x)=T_{m_{k}^{n}} \circ \varphi_{\Delta_{k}, \varepsilon_{k}^{n}}\left(x_{n}, x_{m_{k}^{n}}\right)$ : the function $H: X_{p} \rightarrow X_{q}$ we are looking for is defined by

$$
H(x)=\sum_{n \in \mathbb{N}} \sum_{k \in \mathbb{N}} f_{n, k}(x)
$$


As in the proof of Theorem 5.4, $H$ is well defined, Gâteaux differentiable at each point of $X_{p}$, and Lipschitz continuous, and there exists $a>0$ such that for every $x, y \in X_{p}$, if $x \neq y$, then $\left\|H^{\prime}(x)-H^{\prime}(y)\right\|_{\mathscr{L}\left(\ell^{p}, \ell^{q}\right)} \geq a$.

\section{References}

[1] D. Azagra and R. Deville, James' theorem fails for starlike bodies, J. Funct. Anal. 180 (2001), no. $2,328-346$.

[2] D. Azagra, M. Fabian, and M. Jiménez-Sevilla, Exact filling of figures with the derivatives of smooth mappings between Banach spaces, to appear in Canadian Mathematical Bulletin.

[3] D. Azagra and M. Jiménez-Sevilla, On the size of the sets of gradients of bump functions and starlike bodies on the Hilbert space, Bull. Soc. Math. France 130 (2002), no. 3, 337-347.

[4] D. Azagra, M. Jiménez-Sevilla, and R. Deville, On the range of the derivatives of a smooth function between Banach spaces, Math. Proc. Cambridge Philos. Soc. 134 (2003), no. 1, 163-185.

[5] J. M. Borwein, M. Fabian, I. Kortezov, and P. D. Loewen, The range of the gradient of a continuously differentiable bump, J. Nonlinear Convex Anal. 2 (2001), no. 1, 1-19.

[6] J. M. Borwein, M. Fabian, and P. D. Loewen, The range of the gradient of a Lipschitz $C^{1}$-smooth bump in infinite dimensions, Israel J. Math. 132 (2002), 239-251.

[7] R. Deville and P. Hájek, On the range of the derivative of Gâteaux-smooth functions on separable Banach spaces, Israel J. Math 145 (2005), 257-269.

[8] R. Deville and M. Ivanov, Smooth variational principles with constraints, Arch. Math. (Basel) 69 (1997), no. 5, 418-426.

[9] M. Fabian, O. F. K. Kalenda, and J. Koláŕ, Filling analytic sets by the derivatives of $C^{1}$-smooth bumps, Proc. Amer. Math. Soc. 133 (2005), no. 1, 295-303.

[10] T. Gaspari, On the range of the derivative of a real-valued function with bounded support, Studia Math. 153 (2002), no. 1, 81-99.

[11] Bump functions with Hölder derivatives, Canad. J. Math. 56 (2004), no. 4, 699-715.

[12] P. Hájek, Smooth functions on $c_{0}$, Israel J. Math. 104 (1998), 17-27.

[13] T. Mátrai, Graphs of Gâteaux derivatives are $w^{*}$-connected, Real Anal. Exchange 29 (2003/2004), no. 1, 291-297.

Robert Deville: Laboratoire Bordelais d'Analyse et Geométrie, Institut de Mathématiques de Bordeaux, Université de Bordeaux 1, 351 cours de la Libération, 33405 Talence Cedex, France

E-mail address: deville@math.u-bordeaux.fr 


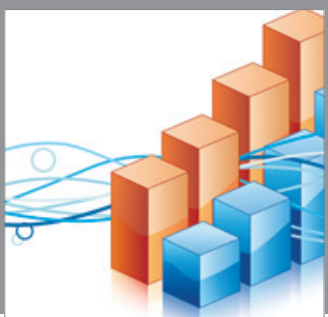

Advances in

Operations Research

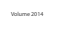

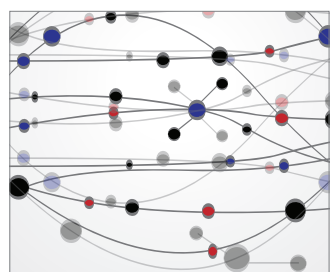

\section{The Scientific} World Journal
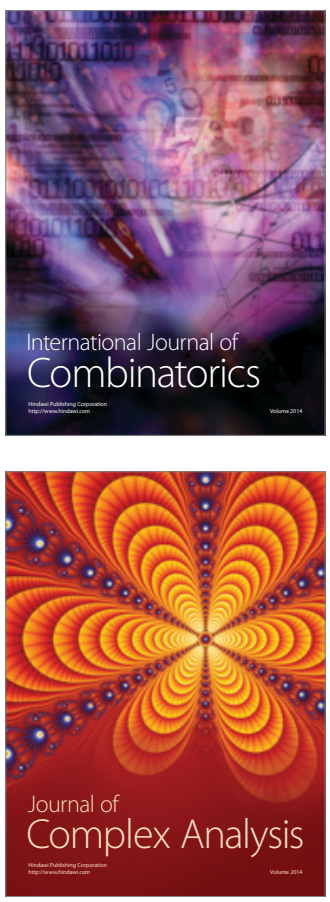

International Journal of

Mathematics and

Mathematical

Sciences
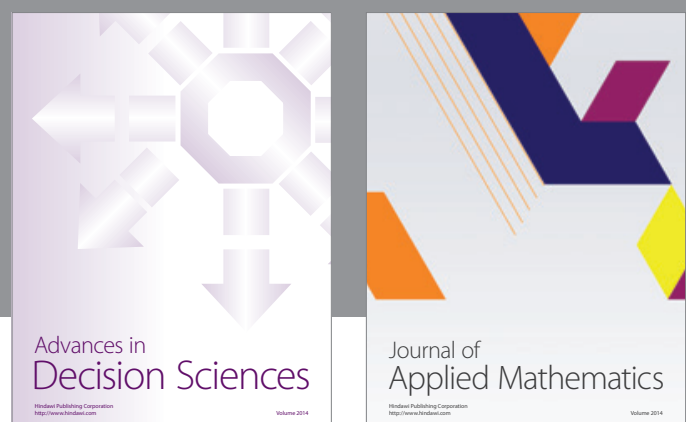

Journal of

Applied Mathematics
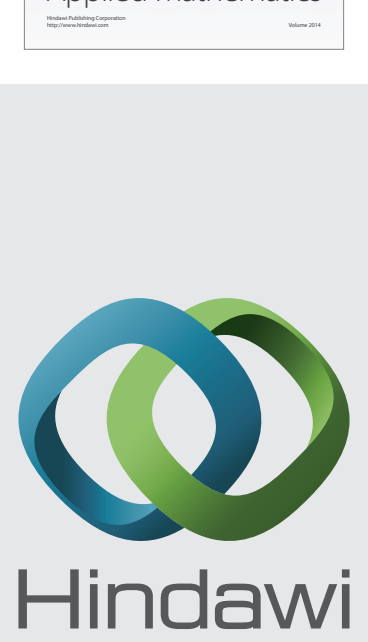

Submit your manuscripts at http://www.hindawi.com
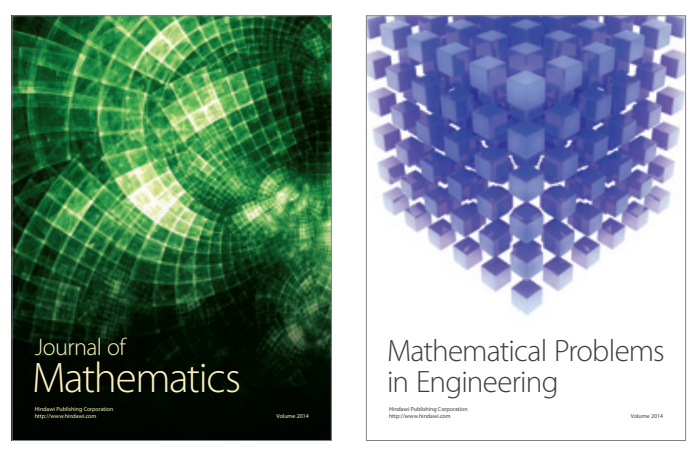

Mathematical Problems in Engineering
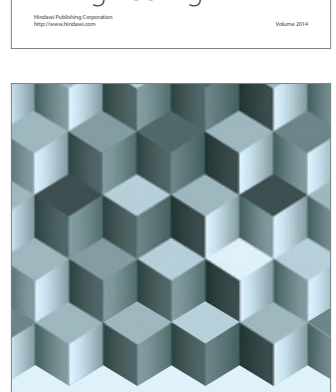

Journal of

Function Spaces
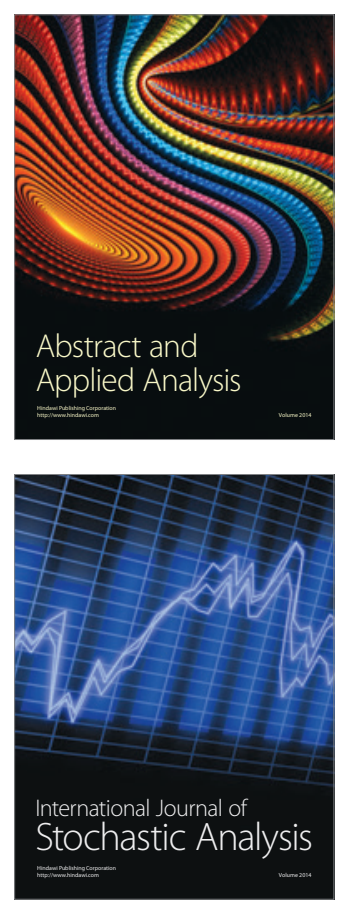

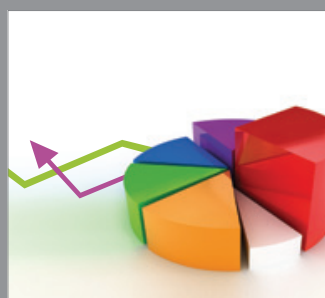

ournal of

Probability and Statistics

Promensencen
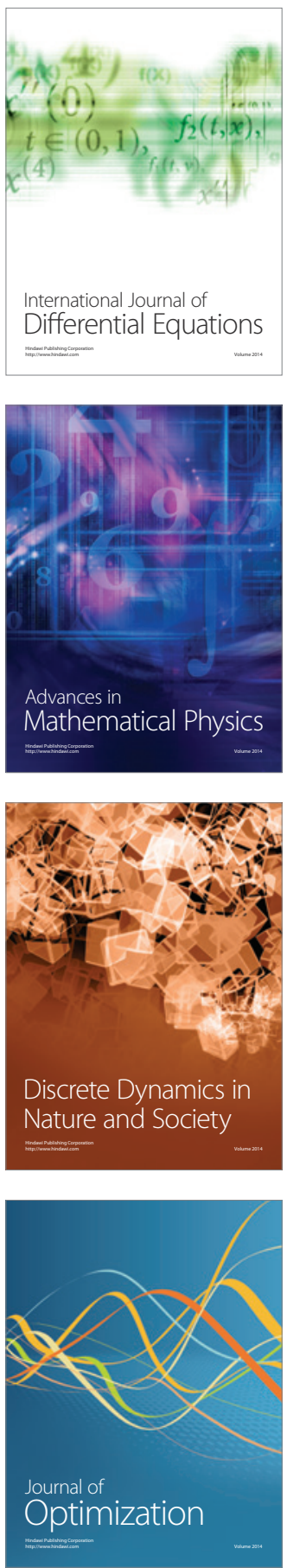